\title{
Open Science and the Role of Cardiology Journals in the COVID-19 Pandemic
}

Claudio Tinoco Mesquita ${ }^{1,2,3}$

Pós-Graduação em Ciências Cardiovasculares, Ebserh/HUAP, Universidade Federal Fluminense, ${ }^{1}$ Niterói, RJ - Brazil

Hospital Pró-Cardíaco, ${ }^{2}$ Rio de janeiro, $R J$ - Brazil

Editor-in-Chief International Journal of Cardiovascular Sciences, Sociedade Brasileira de Cardiologia, ${ }^{3}$ Rio de Janeiro, RJ - Brazil

"It is not the strongest of the species that survives, not the most intelligent that survives.

It is the one that is the most adaptable to change." - Charles Darwin

In July 2020, Brazil has the world's second highest Covid-19 death toll. The COVID-19 pandemic is spreading fast in America. Since the first case of COVID-19 was confirmed, it took 114 days in Brazil (February 26-June 19) and 98 days in the USA (January 21-April 29) for the number of cases to reach more than 1,000,000. Parallel to the rapid growth of COVID-19 cases, there has been a progression in the number of scientific publications. Until June 2020, more than 25,800 papers about COVID-19 were published in PubMed (17,800 open access - 69\%). This volume of scientific publications is unprecedent. The Journal of the American Medical Association (JAMA), for example, received more than 11,000 submissions from January 1 to June 1, 2020, compared with approximately 4,000 submissions during the same period in 2019. ${ }^{1}$ The International Journal of Cardiovascular Sciences is facing a similar situation with more than doubled manuscripts submitted. Our aim is to discuss the importance of Open Science during the COVID-19 pandemic and the role of cardiology journals in this special moment.

COVID-19 is a new disease and many of its aspects are still obscure. What is known about SARS-CoV-2 transmission, incubation, and environmental stability? What are the risk factors for the disease? What is the best

\section{Keywords}

COVID-19; Coronavirus; Pandemics; Scientific Publications; Peer Review; Ethical Review; Open Access Publishing. evidence-based therapy? What is the real importance of asymptomatic and presymptomatic virus shedding in SARS- CoV-2 transmission? How long neutralizing antibodies persist following infection, and do they confer immunity to reinfection? These and many other key questions are still unanswered. ${ }^{2}$ There is a great need for fast and efficient publication of information, but at the same time all efforts must be made to assure quality, and avoid biases and limitations. ${ }^{3}$

Research reports submitted to the IJCS are initially reviewed by the editor-in-chief, then by the associate editor, and finally by reviewers. For manuscripts that need revisions, the entire peer review process takes no less than 60 days. As a task force of the IJCS editors and reviewers, COVID-19 manuscripts are under fast track to reduce the time to response to the authors to 15 days and accelerate the time from submission to publication, and importantly, of reliable data. Our first fast-track paper about COVID-19 was published in April covering the cardiovascular consequences of SARS-CoV-2 infection. ${ }^{4}$ In a two-month period, this article was cited in two papers and the preprint was downloaded more than 400 times. The second fast-track paper was an editorial proposing a framework to fight against fake medical news, which can

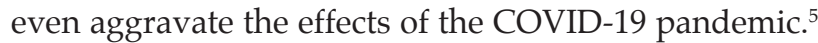
Many articles on COVID-19 are about to be published in the IJCS. What is the impact of this acceleration in the publication process? Horbach studied the duration of publication process in medical journals and found that, compared to prior pandemic, turnaround times have decreased on average by $49 \%$ during the pandemic, and publication process became nearly twice as fast for Covid-19 related articles. ${ }^{6}$

Peer review is essential in science and editors must assure scientific rigor in methodological issues and solid statistical analyses. ${ }^{1}$ Scientific misconduct (fabrication,

Mailing Address: Claudio Tinoco Mesquita

Universidade Federal Fluminense Faculdade de Medicina - Departamento de Radiologia - Av. Marques do Paraná, 303. Postal Code: 24230-322, Centro,

Niterói, RJ - Brazil

E-mail: claudiotinocomesquita@id.uff.br

DOI: https://doi.org/10.36660/ijcs.20200191 
falsification, and plagiarism) is directly related to the urge to publish more $^{7}$ and has affected prestigious medical journals since the beginning of the pandemic. The New England Journal of Medicine and The Lancet are among the oldest, most respected and most influential medical journals in the world. Both journals had important COVID-19 papers ${ }^{8,9}$ retracted due to data fabrication. Most of the time, reviewers do not examine the raw data of the studies they review. One of the multiple benefits of Open Science is that research data can be checked by anyone who accesses the data repository, thereby reducing the likelihood of scientific misconduct. ${ }^{10}$

What is the role of cardiology journals during COVID-19 pandemic? First, they must adapt to the urgent needs of fast peer review and editorial evaluation. Second, cardiology journals must ensure scientific rigor and

\section{References}

1. Bauchner H, Fontanarosa PB, Golub RM. Editorial Evaluation and peer review during a pandemic. JAMA.2020 June 26;(online). Doi: 10.1001/ jama.2020.11764

2. Yuen KS, Ye ZW, Fung SY, Chan CP, JinDY. SARS-CoV-2 and COVID-19: the most important research questions. Cell Biosci.2020;10(40):1-5.

3. Moreira LFP. The importance of scientific publications in times of pandemic crisis. Clinics. 2020;76 Doi: 10.6061/clinics/2020/e1895 bg

4. Oliveira GMM, Pinto FJ. COVID-19: a matter close to the heart, Int J Cardiovasc Sci. 2020;33(3):199-202.

5. Mesquita CT, Oliveira A, Seixas FL, Paes A. Infodemia, fake news and medicine : sciece and the quest for truth. Int J Cardiovasc Sci. 2020;33(3):203-5.

6. Horbach SPJM.Pandemic publishing medical journals drastically speed up their publication process for COVID-1. bioRxiv Doi:10.1101/2020.04.18.045963 research integrity. Third, they must focus on the cardiac aspects of COVID-19 because the cardiology community needs reliable resources of specific information related to their practice, such as the influence of previous heart conditions, safe cardiological practices, cardiac effects of COVID-19 therapy, typical cardiac manifestations of COVID-19, the effects of quarantine on the cardiovascular system and many other emerging issues. Fourth, and finally, non-COVID-19 cardiac research must not be forgotten. All cardiac diseases and their consequences still exist, and many unmet needs of non-COVID-19 cardiac diseases must be pursued. Science must provide answers for these and many other questions. Cardiology journals must accomplish their mission and provide their readers with comprehensive knowledge on cardiovascular sciences in the best way possible.

7. Mesquita CT. Integrity in Scientific Research. Int J Cardiovasc Sci. 2017;30(1):1-3.

8. Mehra MR, Desai SS, Ruschitzka F, Patel NA. Hydroxychloroquine or chloroquine with or without a macrolide for treatment of COVID-19: a multinational registry analysis. Lancet. 2020 May 22. Doi: 10.016/S01406736(20)31180-6

9. Mehra MR, Desai SS, Kuy S, Henry TD, Patel NA. Cardiovascular disease,drug therapy and mortality in COVID-19. N Engl J Med. 2020 Jun 18;382(25):e102. Doi: 10.1056/NEJMoa2007621. Epub 2020,May1

10. Mesquita CT, Borim D, Rochitte CE. Open science,cardiology and 20 years of SciELO (Scientific Electronic Library Online) Int J Cardiovasc Sci. 2019;32(3):203-4. 